\title{
A frequent gene polymorphism affecting the heart-rate response to carvedilol
}

"...carvedilol may be not the best suited $\beta$-blocker for patients in whom rate control in chronic AF is a primary goal or it should be given at high doses."

\section{KEYWORDS: $\beta$-blockers $\approx A D B R 1$ gene polymorphisms $\approx$ atrial fibrillation $\approx$ bisoprolol - carvedilol $=$ heart failure}

$\beta$-adrenoceptor antagonists, known as $\beta$-blockers, are a cornerstone in the treatment of the most prevalent cardiovascular syndromes, including heart failure, ischemic heart disease, atrial fibrillation and hypertension. Their beneficial effects are explained by competitive antagonism at $\beta 1$-adrenoceptors, the dominant adrenergic receptors expressed in cardiac myocytes. By contrast, antagonism at $\beta 2$-adrenoceptors accounts for side effects (e.g., vasospasm ['cold extremities'], bronchospasm and hypoglycemia) and contraindications, such as asthma bronchiale. Accordingly, the majority of currently prescribed second-generation $\beta$-blockers (e.g., metoprolol, bisoprolol and atenolol) preferentially act at $\beta 1$-adrenoceptors, that is, they possess higher affinity towards $\beta 1$ - than $\beta 2$-adrenoceptors (' $\beta 1$-selective $\beta$-blockers'). Carvedilol is a thirdgeneration $\beta$-blocker, which is generally classified as nonselective, but, in fact, has a 13-fold-higher affinity for human $\beta 2$ - than $\beta 1$-adrenoceptors [1]. Carvedilol is also a potent $\alpha 1$-adrenoceptor antagonist [2], which balances the constrictive effect of $\beta 2$-blockade, and has antioxidant effects of unknown relevance. Moreover, carvedilol directly blocks spontaneous diastolic $\mathrm{Ca}^{2+}$ release from cardiac ryanodine receptors, an activity thought to suppress ventricular arrhythmias, for example in heart failure, where the ryanodine receptor is thought to be 'leaky' [3,4]. Finally, carvedilol has unusually slow dissociation kinetics, that is, it 'sticks to adrenoceptors', which causes a longer time of action than predicted from its plasma half-life [5].

The $\beta 1$-adrenoceptor gene (ADBR1) exhibits a frequent SNP that causes a change of Arg at position 389 to Gly [6]. Approximately 40 and 7\% of Caucasians are heterozygous and homozygous for the rarer Gly389 variant, respectively. The polymorphism has gained much attention because, when overexpressed at very high levels in heterologous cell systems, the Gly389 variant stimulates cAMP synthesis fivefold less than the Arg389 variant, that is, it is a minus variant. Since chronic hyperactivity of $\beta 1$-adrenoceptors is thought to adversely affect cardiovascular outcome and $\beta$-blockade improves it $[7,8]$, it was initially assumed that the homozygous presence of the Arg389 variant would confer a higher risk in patients at high cardiac risk, on the one hand, and better response to $\beta$-blockade on the other hand. Indeed, in retrospective clinical analyses, some studies in transgenic mice and isolated human heart muscle preparations reported higher signaling activity of the Arg389 variant and greater responses to $\beta$-blockade (see overview in $[9,10])$. The only large prospective outcome study found no difference between homozygous Arg389 and Gly/X carriers in the placebo group, but benefit from the atypical $\beta$-blocker bucindolol only in the Arg389Arg group [11]. Several other studies failed to substantiate differences between the groups.

On this background, we performed a prespecified pharmacogenetics trial accompanying the CIBIS-ELD trial. CIBIS-ELD prospectively evaluated the tolerability of the two standard $\beta$-blockers carvedilol and bisoprolol in $>800$ elderly patients with heart failure, a group prone to side effects to $\beta$-blockers. The main findings of CIBIS-ELD were: using a 2-weekly uptitration scheme, only approximately $25 \%$ of the elderly patients with heart failure reached the respective guideline target dose; and bisoprolol caused greater heart-rate reductions and more bradycardia than carvedilol [12]. The hypothesis of the pharmacogenetic substudy of CIBIS-ELD was based on the above biochemical evidence that the Gly389 $\beta 1$-adrenoceptor is a minus variant and stated that Arg389Arg carriers have a greater heart-rate response to $\beta$-blockers. The results in $>500$ patients available for the genetic

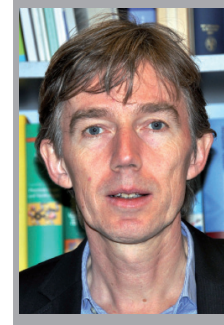

Thomas Eschenhagen

Department of Experimental Pharmacology \& Toxicology, University Medical Center Hamburg Eppendorf, Hamburg, Germany and DZHK (German Centre for Cardiovascular Research), Partner Site Hamburg/Kiel/Lübeck, Germany t.eschenhagen@uke.uni-hamburg.de 
analysis were surprising and did not support our hypothesis (Figure 1) [9]. Predrug heart rate did not differ between the genotypes, neither in patients with sinus rhythm (SR) nor with atrial fibrillation (AF; $20 \%$ of total cohort) [7]. In patients with $\mathrm{SR}$, neither the genotype nor the type of $\beta$-blocker affected the heart-rate response to $\beta$-blockade (reduction from $\sim 70$ to $64 \mathrm{bpm}$ ) [3]. By contrast, patients with AF showed a smaller heart-rate response to carvedilol than to bisoprolol. The difference was entirely due to a markedly smaller effect of carvedilol in Arg389Arg carriers, whereas the effect of carvedilol and bisoprolol did not differ in Gly/X carriers [13]. Surprisingly, the differences between genotypes and $\beta$-blockers emerged only after 2 weeks of treatment. Acute responses were identical in all groups. Thus, the study identified a remarkable fading of bradycardic effects of carvedilol only in heart failure patients in AF that carry two alleles encoding the active $\beta 1$-adrenoceptor variant Arg389.

\section{Is it a true pharmacogenetic effect?}

It could be argued that the results came along just by chance, adding to the noise in this scientific field. Arguments against this explanation are the relatively large number of patients studied in a randomized, prospective and double-blinded manner [9], the highly statistically significant effects (differences between genotypes and between carvedilol and bisoprolol $\mathrm{p}<0.0001$ ) [7], and the fact that the data are well in line with two other studies [3]. A register study in Japanese patients with heart failure reported the heart-rate-lowering effect of carvedilol to be smaller than that of bisoprolol, both in the entire cohort and the AF subgroup [14]. Also, a recent nonrandomized cohort study in patients with AF observed that the heart-rate-lowering effect of $\beta$-blockers in general was greater in Gly389 carriers [15]. In line with our data, the difference was most pronounced with carvedilol. Bisoprolol was not studied. Taken together, the difference in heart-rate response between Gly389 and Arg389Arg carriers is to date one of the best documented, independently reproduced and most pronounced pharmacogenetic effect in the cardiovascular field.

\section{Mechanisms?}

The mechanisms of the difference between genotypes, $\beta$-blockers, SR and AF, and acute and prolonged effects are currently unclear. Several self-evident explanations seem unlikely. The higher signaling activity of the Arg389 variant would have predicted a stronger response (to both $\beta$-blockers). The fact that basal heart rate and the response to $\beta$-blockers were essentially identical in patients with SR (and acutely in all groups) therefore suggests that compensatory mechanisms are in place that counteract the higher signal input through the Arg389 variant. The $\alpha 1$-adrenoceptor antagonist and vasodilating property of carvedilol predicts its blood pressure-lowering effect to be larger than that of bisoprolol. This could cause reflex sympathetic nervous activation via baroreceptors and therefore minimize the heart-rate-lowering effect of carvedilol. However, such an indirect effect would be seen acutely and in all patients, which was not the case. Instead, the data show slowly developing genotype-specific regulation of atrioventricular node (AVN) conduction, but not of pacemaking in the sinoatrial node. Catecholamines accelerate AVN conduction by stimulating the main depolarizing inward current, $\mathrm{I}_{\mathrm{Ca}}$, and shorten the effective refractory period by stimulating $\mathrm{I}_{\mathrm{Kur}}$ (see review in [16]). $\beta$-blockers antagonize these effects and thereby slow AVN conduction velocity. In AF, they slow ventricular rate mainly by prolonging the effective refractory period and thereby increasing the filter function of the AVN. In dogs, both $\beta 1$ - and $\beta 2$-adrenoceptors participate in the acceleration of conduction induced by sympathetic nerve stimulation, but the contribution of $\beta 2$-adrenoceptors was smaller in the AVN than the sinoatrial node [17]. Since carvedilol is a preferential $\beta 2$-blocker, one could hypothesize that it is simply not effective enough at low doses to block $\beta 1$-adrenoceptors in the AVN. Why does the difference then develop only after 2 weeks and is seen only in Arg389Arg carriers? It is known that $\beta$-adrenoceptors are upregulated under chronic $\beta$-blockade and it is possible that upregulation of the higher activity Arg389 variant, but not the lower Gly389 variant, overcomes the effect of carvedilol. Alternatively, the observed long-term pharmacogenetic differences in the carvedilol response may be related to the fact that carvedilol is a 'biased agonist' at $\beta$-adrenoceptors. Carvedilol can activate arrestin-dependent, nonclassic, $\beta 1$-dependent signal pathways, such as activation and internalization of EGFRs with subsequent phosphorylation of MAPKs [18]. This may somehow affect its effect on conduction. Obviously, more work is needed to answer this question.

\section{Practical consequences?}

The data show that Arg389Arg carriers with heart failure and AF ( $50 \%$ of patients with $\mathrm{AF}$ and $10 \%$ of all patients with heart failure) 


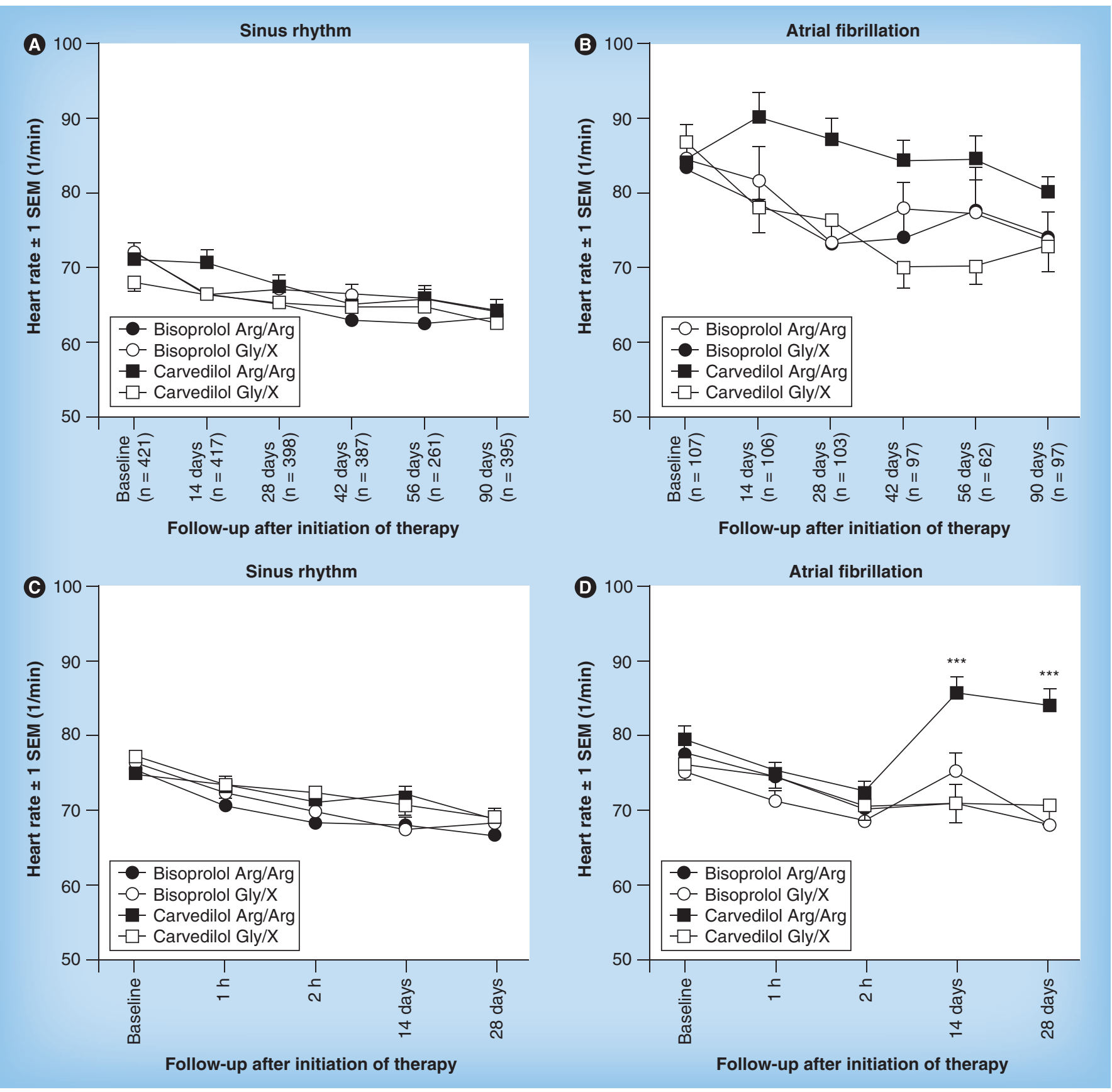

Figure 1. Heart-rate response to uptitration with the $\beta$-blockers bisoprolol $(1.25-10 \mathrm{mg})$ or carvedilol ( $2 \times 3.125-25 \mathrm{mg})$ in patients with chronic heart failure according to rhythm and $\beta 1$-adrenoceptor genotype (Arg389Gly). (A \& B) Resting heart rate at baseline (predrug), 14 (bisoprolol: $1.25 \mathrm{mg}$; carvedilol: $2 \times 3.125 \mathrm{mg}$ ), 28 (bisoprolol: $2.5 \mathrm{mg}$; carvedilol: $2 \times 6.25 \mathrm{mg}$ ), 42 (bisoprolol: $5 \mathrm{mg}$; carvedilol: $2 \times 12.5 \mathrm{mg}$ ), 56 (bisoprolol: $10 \mathrm{mg}$; carvedilol: $2 \times 25 \mathrm{mg}$ ) and 90 days (follow-up) after initiation of $\beta$-blocker therapy in patients with (A) sinus rhythm or (B) atrial fibrillation. Numbers in brackets indicate numbers of patients at the respective time points/dose level. (C \& D) Resting heart rate at baseline (predrug), 1, 2 and 14 days after initiation of $\beta$-blocker treatment with the starting dose (bisoprolol: $1.25 \mathrm{mg}$; carvedilol: $3.125 \mathrm{mg}$ ) and after 28 days (bisoprolol: $2.5 \mathrm{mg}$; carvedilol: $2 \times 6.25 \mathrm{mg}$ ) in patients with (C) sinus rhythm or (D) atrial fibrillation. Note that $25 \%$ of patients were pretreated with a $\beta$-blocker at $<25 \%$ of target dose, likely explaining why carvedilol formally caused an increase in mean heart rate at 14 and 28 days after initiation of treatment in patients with atrial fibrillation ((B) and (D), respectively).

$* * * p<0.0001$ versus bisoprolol or Gly/X.

Adapted with permission from [12].

lack a sustained heart-rate-lowering response to medium doses of carvedilol. Whereas our own study was restricted to patients with heart failure, the recently published cohort study [15] included all patients with AF and came to similar conclusions, suggesting that the reduced heartrate response to carvedilol pertains to all patients with AF. It is important to note that neither study 
measured effects on outcome. In fact, beneficial effects of $\beta$-blockers on outcome in patients with heart failure and AF have never been prospectively studied. Retrospective analyses of large-outcome studies suggested significant benefits of treatment with carvedilol [13] and formally not with bisoprolol [19] or metoprolol [20]. On the other hand, a retrospective study in patients with heart failure observed an association of the Arg389Arg (plus Gln27/X for the $\beta 2$-adrenoceptor) genotype with a twofold higher mortality risk in patients treated with carvedilol, but not with metoprolol [21]. Despite small numbers and the retrospective design, these data point to a smaller effect of carvedilol in patients with specific $\beta$-adrenoceptor genotypes.
Clearly, more work is needed to substantiate this hypothesis. In any case, carvedilol may be not the best suited $\beta$-blocker for patients in whom rate control in chronic AF is a primary goal or it should be given at high doses.

\section{Financial \& competing interests disclosure}

The author has no relevant affiliations or financial involvement with any organization or entity with a financial interest in or financial conflict with the subject matter or materials discussed in the manuscript. This includes employment, consultancies, honoraria, stock ownership or options, expert testimony, grants or patents received or pending, or royalties.

No writing assistance was utilized in the production of this manuscript.

\section{References}

1 Brodde OE. $\beta-1$ and $\beta-2$ adrenoceptor polymorphisms: functional importance, impact on cardiovascular diseases and drug responses. Pharmacol. Ther. 117, 1-29 (2008).

Eschenhagen T. Beta-adrenergic signaling in heart failure adapt or die. Nat. Med. 14, 485-487 (2008).

3 Eschenhagen T. Is ryanodine receptor phosphorylation key to the fight or flight response and heart failure? J. Clin. Invest. 120, 4197-4203 (2010).

4 Joglar JA, Acusta AP, Shusterman NH et al. Effect of carvedilol on survival and hemodynamics in patients with atrial fibrillation and left ventricular dysfunction: retrospective analysis of the US carvedilol heart failure trials program. Am. Heart J. 142, 498-501 (2001).

5 Kawada T, Ishibashi T, Nakazawa M, Satoh S, Imai S. Adrenoceptor-blocking activity and cardiohemodynamic effects of carvedilol in animals. J. Cardiovasc. Pharmacol. 16, 147-153 (1990).

6 Kindermann M, Maack C, Schaller S et al. Carvedilol but not metoprolol reduces betaadrenergic responsiveness after complete elimination from plasma in vivo. Circulation 109, 3182-3190 (2004).

7 Konishi M, Haraguchi G, Kimura S et al. Comparative effects of carvedilol vs bisoprolol for severe congestive heart failure. Circ. J. 74, 1127-1134 (2010).
8 Lechat P, Hulot JS, Escolano S et al. Heart rate and cardiac rhythm relationships with bisoprolol benefit in chronic heart failure in CIBIS II Trial. Circulation 103, 1428-1433 (2001).

9 Liggett SB, Mialet-Perez J, Thaneemit-Chen S et al. A polymorphism within a conserved $\beta 1$-adrenergic receptor motif alters cardiac function and beta-blocker response in human heart failure. Proc. Natl Acad. Sci. USA 103 , 11288-11293 (2006).

10 Lohse MJ, Engelhardt S, Eschenhagen T. What is the role of beta-adrenergic signaling in heart failure? Circ. Res. 93, 896-906 (2003).

11 Marx SO, Reiken S, Hisamatsu Y et al. PKA phosphorylation dissociates FKBP12.6 from the calcium release channel (ryanodine receptor): defective regulation in failing hearts. Cell 101, 365-376 (2000).

12 Mason DA, Moore JD, Green SA, Liggett SB. A gain-of-function polymorphism in a G-protein coupling domain of the human $\beta 1$-adrenergic receptor. J. Biol. Chem. 274, 12670-12674 (1999).

13 Molenaar P, Christ T, Ravens U, Kaumann A. Carvedilol blocks $\beta 2$ - more than $\beta 1$ adrenoceptors in human heart. Cardiovasc. Res. 69, 128-139 (2006).

14 Parvez B, Chopra N Rowan S et al. A common $\beta 1$-adrenergic receptor polymorphism predicts favorable response to rate-control therapy in atrial fibrillation. J. Am. Coll. Cardiol. 59, 49-56 (2012)
15 Petersen M, Andersen JT, Hjelvang BR et al. Association of beta-adrenergic receptor polymorphisms and mortality in carvediloltreated chronic heart-failure patients. Br. J. Clin. Pharmacol. 71, 556-565 (2011).

16 Rau T, Dungen HD, Edelmann F et al. Impact of the $\beta 1$-adrenoceptor Arg389Gly polymorphism on heart-rate responses to bisoprolol and carvedilol in heart-failure patients. Clin. Pharmacol. Ther. 92, 21-28 (2012).

17 Takei M, Furukawa Y, Narita M et al. Sympathetic nerve stimulation activates both $\beta 1$ - and $\beta 2$-adrenoceptors of SA and AV nodes in anesthetized dog hearts. Jpn J. Pharmacol. 59, 23-30 (1992).

18 Taylor MR. Pharmacogenetics of the human $\beta$-adrenergic receptors. Pharmacogenomics J. 7, 29-37 (2007).

19 van Veldhuisen DJ, Aass H, El Allaf D et al. Presence and development of atrial fibrillation in chronic heart failure. Experiences from the MERIT-HF Study. Eur. J. Heart Fail. 8, 539-546 (2006).

20 Wisler JW, DeWire SM, Whalen EJ et al. A unique mechanism of beta-blocker action: carvedilol stimulates beta-arrestin signaling. Proc. Natl Acad. Sci. USA 104, 16657-16662 (2007).

21 Workman AJ. Cardiac adrenergic control and atrial fibrillation. Naunyn Schmiedebergs Arch. Pharmacol. 381, 235-249 (2010). 